\title{
Polarization correlation in the two-photon decay of atomic hydrogen: nonlocality versus entanglement
}

\author{
T. Radtke ${ }^{1, a}$, A. Surzhykov ${ }^{2, b}$, and S. Fritzsche ${ }^{3,4}$ \\ 1 Institut für Physik, Universität Kassel, 34132 Kassel, Germany \\ 2 Physikalisches Institut, Universität Heidelberg, 69120 Heidelberg, Germany \\ 3 Max-Planck-Institut für Kernphysik, 69029 Heidelberg, Germany \\ ${ }^{4}$ Gesellschaft für Schwerionenforschung (GSI), 64291 Darmstadt, Germany
}

Received 25 April 2008

Published online 11 July 2008 - (c) EDP Sciences, Società Italiana di Fisica, Springer-Verlag 2008

\begin{abstract}
From the work by Perrie et al. [Phys. Rev. Lett. 54, 1790 (1985)], photon pairs from the $2 s_{1 / 2} \rightarrow 1 s_{1 / 2}$ (two-photon) decay of atomic hydrogen are known to be quantum mechanically correlated. In these experiments, the polarization states of the photons emitted in back-to-back geometry were shown to violate the Bell inequality as a qualitative sign of nonlocality and entanglement. In the present contribution, we analyze how these nonlocal quantum correlations, as given by the violation of the Bell inequality, differ from the concurrence as a true entanglement measure. Results are shown for both quantifiers in dependence of the decay geometry and the initial polarization of the atoms for the $2 s_{1 / 2} \rightarrow 1 s_{1 / 2}$ and $3 d_{5 / 2} \rightarrow$ $1 s_{1 / 2}$ two-photon decay of atomic hydrogen. These results display the difference between nonlocality and entanglement and, hence, may stimulate further experiments on nonlocal quantum correlations in atomic systems.
\end{abstract}

PACS. 32.10.-f Properties of atoms - 31.10.+z Theory of electronic structure, electronic transitions, and chemical binding - 03.67.Bg Entanglement production and manipulation - 03.65.Ud Entanglement and quantum nonlocality

\section{Introduction}

Since the foundation of quantum mechanics, the two-photon transitions in the hydrogen atom have been the subject of intense studies both in theory and experiment $[1-10]$. While, initially, the main emphasis was placed on the total decay rates and energy distribution of the emitted photon pairs, recently the correlations in the polarization state of the $2 s_{1 / 2} \rightarrow 1 s_{1 / 2}$ photons have been analyzed in detail [11-13], following the pioneering experiments by Aspect et al. [14]. In these experiments, it was demonstrated that the polarization correlation cannot be explained by any local realistic theory that uses hidden variables. This experimental test was based on the seminal work of Bell [15] who derived an inequality that limits the correlation of two measurement outcomes if one assumes that the measured observables correspond to local statistical variables.

Indeed, the measured photon polarization correlations in [14] clearly violated the Bell inequality, i.e., they were stronger than any local hidden variable theory admitted. Therefore, the experiment ruled out any chance of de-

\footnotetext{
a e-mail: tradtke@physik.uni-kassel.de

b e-mail: surz@physi.uni-heidelberg.de
}

scribing quantum mechanics using such a hidden variable theory. The experimental evidence that nonlocality is indeed an intrinsic feature of quantum mechanics is well in contrast to Einstein's beliefs in his historical debate with Bohr and Schrödinger who introduced the notion of entanglement for nonproduct (pure) states [16-18]. The application of these intriguing nonclassical features of quantum mechanics has been investigated extensively during the past decade in the context of quantum information theory where it was shown that entanglement is the crucial resource in quantum information protocols such as teleportation, quantum cryptography or efficient quantum computing algorithms that can outperform their classical counterparts [19]. Since then the quantification of entanglement has remained as a central problem. These investigations (see Ref. [20] for a recent review on entanglement measures) also showed that, in general, one needs to distinguish between nonlocality (as indicated by the violation of Bell's inequality) and entanglement as the formal nonseparability of a composite (possibly mixed) quantum state as defined in the famous work of Werner [21].

In this work, we reconsider the two-photon decay of hydrogen in view of the polarization correlation between the emitted photons. To this end, the violation of the CHSH inequality due to Clauser et al. [22], i.e. a particular 
variant of Bell's inequality, is compared with the (socalled) concurrence measure of entanglement [23], based on the reduced density matrix of the photon pair. In the next section, we briefly summarize the calculation of the final two-photon density matrix by means of the relativistic Green's function approach and also introduce (in Sect. 2.3) the measures for the Bell violation and entanglement. In Section 3, we later present the results for the $2 s_{1 / 2} \rightarrow 1 s_{1 / 2}$ and $3 d_{5 / 2} \rightarrow 1 s_{1 / 2}$ decay of atomic hydrogen for different decay geometries and polarization (sublevel population) of the initial state. Finally, a brief summary is given in Section 4 .

\section{Theoretical background}

Not much need to be said about the basic formalism for studying the two-photon emission from one-electron atoms. In the past, this formalism has been applied widely in order to explore not only the total decay rates [5] but also the energy as well as angular distributions [7,24,25] and even the correlation in the polarization state of the photons [26]. Below, we therefore restrict ourselves to a rather short account of the basic expressions, just enough for introducing the quantum measures for the nonlocality and nonseparability of the state of the photon pair.

\subsection{Choice of coordinates}

To analyze the (correlated) spin state of two photons quantitatively, we need to introduce proper coordinates. Let us suppose that the excited atom is either aligned or polarized before its decay along the $z$-axis, which is used for quantization. Together with the direction of the first photon $\boldsymbol{k}_{1}$, this quantization axis then defines the reaction plane $(x-z$ plane $)$ and only one polar angle $\theta_{1}$ is required to characterize the first decay photon, while the two angles $\theta_{2}$ and $\phi_{2}$ are utilized to specify the emission of the second photon (see Fig. 1).

This 'geometry' for the emission of the photon pair becomes even simpler if the excited atomic state is initially unaligned (unpolarized). In this case, there is no distinguished direction for the system and, hence, it is convenient to adopt the $z$-axis along the momentum of the first photon $\boldsymbol{k}_{1}$. The opening angle $\theta \equiv \theta_{2}$ between the two photons is then sufficient to characterize the decay geometry completely.

\subsection{Two-photon density matrix}

Most naturally, the spin states of the emitted photons are described within the density matrix approach [27-29]. Having defined the 'geometry' in the last subsection, the two-photon density matrix for an atomic transition from the initial state $\left|n_{i} \kappa_{i}\right\rangle$ to the final state $\left|n_{f} \kappa_{f}\right\rangle$ is given

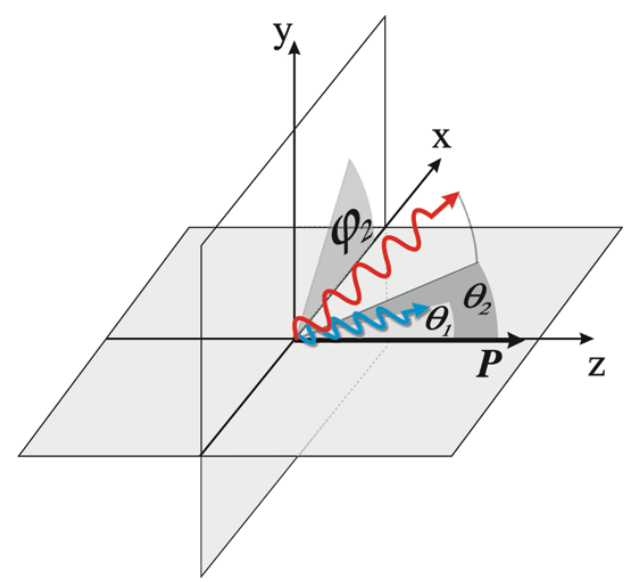

Fig. 1. (Color online) Geometry for the two-photon decay of hydrogen. The quantization axis ( $z$-axis) is defined by the polarization axis $\boldsymbol{P}$ of the atom and gives rise, together with the emission of the first photon under the polar angle $\theta_{1}$, to the reaction plane ( $x-z$ plane). The two angles $\theta_{2}$ and $\varphi_{2}$ are needed then to specify the emission of the second photon.

within the helicity representation by $[26,30]$ :

$$
\begin{array}{r}
\left\langle\boldsymbol{k}_{1} \lambda_{1}, \boldsymbol{k}_{2} \lambda_{2}\left|\hat{\rho}_{\gamma}\right| \boldsymbol{k}_{1} \lambda_{1}^{\prime}, \boldsymbol{k}_{2} \lambda_{2}^{\prime}\right\rangle=\sum_{\mu_{i}, \mu_{i}^{\prime}, \mu_{f}} M_{f i}\left(\mu_{f}, \mu_{i}, \lambda_{1}, \lambda_{2}\right) \\
\times\left\langle n_{i} \kappa_{i} \mu_{i}\left|\hat{\rho}_{i}\right| n_{i} \kappa_{i} \mu_{i}^{\prime}\right\rangle M_{f i}^{*}\left(\mu_{f}, \mu_{i}^{\prime}, \lambda_{1}^{\prime}, \lambda_{2}^{\prime}\right),(1)
\end{array}
$$

where $\boldsymbol{k}_{1,2}$ denotes the wave vector and $\lambda_{1,2}= \pm 1$ the helicity of the photons, that is the spin projection onto their direction of propagation. In the helicity representation, the two spin projections $\lambda= \pm 1$ correspond to the right and left circular polarization states $\left|\sigma^{ \pm}\right\rangle$of a photon.

Equation (1) shows explicitly that the spin state of the emitted photons depends on the population of the excited atomic state, as seen from the density matrix $\hat{\rho}_{i}$, as well as on the transition amplitude $M_{f i}\left(\mu_{f}, \mu_{i}, \lambda_{1}, \lambda_{2}\right)$. This amplitude 'connects' the two bound states $\left|n_{i} \kappa_{i}\right\rangle$ and $\left|n_{f} \kappa_{f}\right\rangle$ of an atom due to the coupling of the electron to the light field and, within the second-order perturbation theory, is given by:

$$
\begin{aligned}
M_{f i}\left(\mu_{f},\right. & \left.\mu_{i}, \lambda_{1}, \lambda_{2}\right)= \\
& \sum_{\nu} \frac{\left\langle\psi_{n_{f} j_{f} \mu_{f}}\left|\boldsymbol{A}_{1}^{*}\right| \psi_{\nu}\right\rangle\left\langle\psi_{\nu}\left|\boldsymbol{A}_{2}^{*}\right| \psi_{n_{i} j_{i} \mu_{i}}\right\rangle}{E_{\nu}-E_{i}+E_{\gamma_{2}}} \\
& +\sum_{\nu} \frac{\left\langle\psi_{n_{f} j_{f} \mu_{f}}\left|\boldsymbol{A}_{2}^{*}\right| \psi_{\nu}\right\rangle\left\langle\psi_{\nu}\left|\boldsymbol{A}_{1}^{*}\right| \psi_{n_{i} j_{i} \mu_{i}}\right\rangle}{E_{\nu}-E_{i}+E_{\gamma_{1}}}
\end{aligned}
$$

In this formula, the operator $\boldsymbol{A}_{i}=\boldsymbol{\alpha} \cdot \boldsymbol{u}_{\lambda_{i}} \mathrm{e}^{\boldsymbol{k}_{i} \cdot \boldsymbol{r}}$ describes the (relativistic) electron-photon interaction and the unit vectors $\boldsymbol{u}_{\lambda_{1,2}}$ the polarization of the individual photons. Since the relativistic second-order transition amplitude has been applied very frequently in studying the twophoton processes, here we shall not discuss the further evaluation of equation (2) and refer the reader for all details to references $[5,7,24,25]$. 


\subsection{Quantum measures to characterize the nonlocality and entanglement of photon pairs}

The density matrix (1) can be utilized for a quantitative analysis of the polarization correlations between the two photons. For this purpose, let us introduce here two measures to quantify the (amount of) entanglement and nonlocality for a given photon pair. Formally, of course, we may identify the polarization of a photon as a qubit (twolevel system) and make use of some well-known results from quantum information theory.

Although the quantification of entanglement is still an open problem for general $N$-qubit systems, Wootter's concurrence measure [23] provides a analytical formula that can be applied to any two-qubit system, such as the polarization state of a photon pair, and that has been widely used. For any two-qubit state $\hat{\rho}$, either pure or mixed, the concurrence is defined as

$$
\mathcal{C}(\hat{\rho})=\max \left(0, \sqrt{e_{1}}-\sqrt{e_{2}}-\sqrt{e_{3}}-\sqrt{e_{4}}\right),
$$

where $\sqrt{e_{i}}$ are the descendingly ordered square roots of the eigenvalues of the matrix $\hat{\rho}\left(\hat{\sigma}_{2}^{(1)} \otimes \hat{\sigma}_{2}^{(2)}\right) \hat{\rho}^{*}\left(\hat{\sigma}_{2}^{(1)} \otimes \hat{\sigma}_{2}^{(2)}\right)$, $\hat{\rho}^{*}$ is the complex conjugate of $\hat{\rho}$, and where $\hat{\sigma}_{2}^{(1,2)}$ are the Pauli $\sigma_{y}$ matrices acting on the first and the second qubit, respectively. With this measure, we are able to quantify the purely quantum mechanical correlations between two qubits. Note that this implies that the concurrence measure vanishes only if the two-qubit state is separable, i.e. if the density matrix can be written as a convex combination of product states [21]:

$$
\hat{\rho}=\sum_{i} p_{i}\left|\psi_{i}\right\rangle\left\langle\psi_{i}|\otimes| \phi_{i}\right\rangle\left\langle\phi_{i}\right| \quad \text { with } p_{i}>0, \sum_{i} p_{i}=1 \text {. }
$$

In previous experiments [11-13], the correlation between the emitted photon pairs has been discussed in terms of their 'incompatibility' with local theories of hidden variables, which can be tested by means of Bell's inequality [15]. It is well-known that quantum states that violate the Bell inequality have to be entangled (i.e. are nonseparable) and actually all pure entangled (two-qubit) states $d o$ violate the Bell inequalities [31]. However, it was found that the situation is different for mixed quantum states where some entangled states, for instance the so-called Werner states, do not violate any Bell-type inequality [21]. Therefore, in order to study the difference between nonlocality and entanglement, we adopt below a measure from references $[32,33]$ that quantifies the violation of a widely used variant of the Bell inequality that is due to Clauser et al. [22]. For the case of two qubits in an arbitrary mixed state $\hat{\rho}$, the Bell-CHSH inequality can be written as

$$
\left|\operatorname{Tr}\left(\hat{\rho} \mathcal{B}_{\mathrm{CHSH}}\right)\right| \leq 2,
$$

where $\mathcal{B}_{\mathrm{CHSH}}$ is the Bell operator given as

$$
\mathcal{B}_{\mathrm{CHSH}}=\boldsymbol{a} \cdot \boldsymbol{\sigma}^{(1)} \otimes\left(\boldsymbol{b}+\boldsymbol{b}^{\prime}\right) \cdot \boldsymbol{\sigma}^{(2)}+\boldsymbol{a}^{\prime} \cdot \boldsymbol{\sigma}^{(1)} \otimes\left(\boldsymbol{b}-\boldsymbol{b}^{\prime}\right) \cdot \boldsymbol{\sigma}^{(2)}
$$

with given unit vectors $\boldsymbol{a}, \boldsymbol{a}^{\prime}, \boldsymbol{b}, \boldsymbol{b}^{\prime} \in \mathbb{R}^{3}$, and $\boldsymbol{\sigma}^{(1,2)}=$ $\left(\hat{\sigma}_{1}^{(1,2)}, \hat{\sigma}_{2}^{(1,2)}, \hat{\sigma}_{3}^{(1,2)}\right)$ is the vector of the Pauli spin matrices acting on the first or second qubit, respectively. If, for a given density matrix $\hat{\rho}$, there exist vectors $\boldsymbol{a}, \boldsymbol{a}^{\prime}, \boldsymbol{b}, \boldsymbol{b}^{\prime}$ so that the criterion (5) is not fulfilled, then the state is said to violate the Bell-CHSH inequality. In order to quantify the (maximal) violation, we first rewrite the two-qubit density matrix in terms of the Pauli basis

$$
R_{i j}=\operatorname{Tr}\left(\hat{\rho} \hat{\sigma}_{i}^{(1)} \otimes \hat{\sigma}_{j}^{(2)}\right) .
$$

With this notation, the 'Bell violation' measure is defined as

$$
B(\hat{\rho})=\sqrt{\max \left(0, s_{1}^{2}+s_{2}^{2}-1\right)},
$$

where $s_{1,2}$ are the two largest singular values of the matrix $R_{i j}[33,34]$. The definition (8) has the useful property that it is always equal to the concurrence measure (3) for pure states.

We shall point out, however, that the Bell violation is not a good entanglement measure for general mixed (two-qubit) states where it provides merely a 'hint' on the strength of entanglement. In general, one has to be cautious in relating the Bell violation and the concurrence measure to each other, since the concurrence measures the degree of quantum mechanical correlations while the Bell violation quantifies the degree of nonlocality that is found in the outcome of a measurement. In this sense, the difference between the two measures can be interpreted also as 'hidden nonlocality'. In particular, the Bell violation may vanish for some mixed states that are actually entangled as we shall demonstrate in the next section for the case of photon pairs emitted during the $3 d_{5 / 2} \rightarrow 1 s_{1 / 2}$ decay of hydrogen.

\section{Results and discussion}

Most easily, a comparison between the nonlocality and entanglement of the polarization state of the photons emitted in the (two-photon) decay of atomic hydrogen can be made for the $2 s_{1 / 2} \rightarrow 1 s_{1 / 2}$ and $3 d_{5 / 2} \rightarrow 1 s_{1 / 2}$ transitions. Below, all computations of the transition amplitudes and the quantum measures were carried out by means of the RATIP [35] and FEYNMAN programs [36] that have been developed in our group during the past years.

\subsection{The $2 \mathrm{~s}_{1 / 2} \rightarrow 1 \mathrm{~s}_{1 / 2}$ decay}

For the $2 s_{1 / 2} \rightarrow 1 s_{1 / 2}$ two-photon decay of hydrogen, it is well-known that the two emitted photons violate the BellCHSH inequalities and, hence, are entangled with each other. This has been studied in particular for the case that the two photons were emitted in back-to-back geometry [11], and for which the experimental results were found to agree well with quantum mechanical predictions. In Figure 2, we display the concurrence and the Bell violation measures as a function of the polar angle $\theta_{2}$, while the two other angles in specifying the geometry of the photon pair are kept fixed, $\theta_{1}=0^{\circ}$ and $\varphi_{2}=0^{\circ}$. As seen from this figure, the two measures coincide exactly in this case since the final polarization state of the photons 


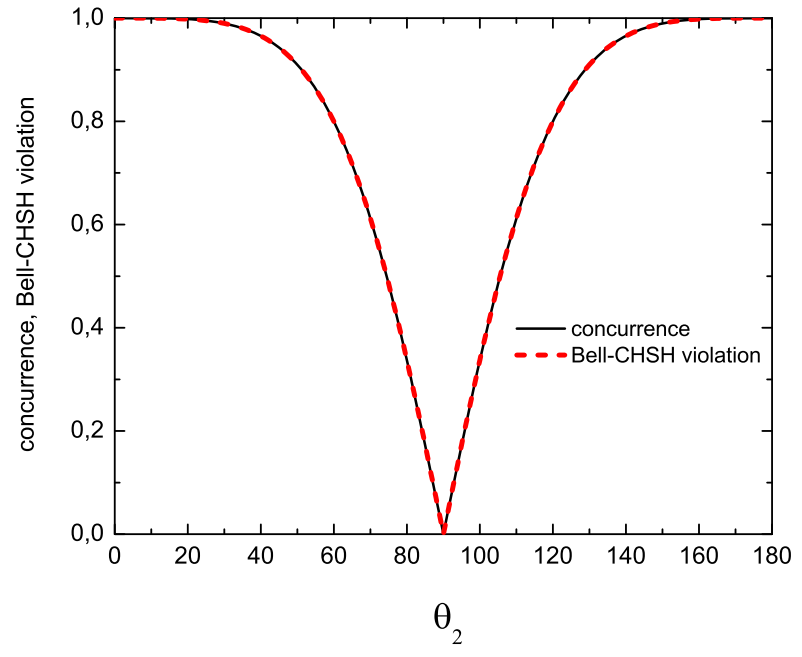

Fig. 2. (Color online) Two-photon polarization entanglement and Bell violation for the $2 s_{1 / 2} \rightarrow 1 s_{1 / 2}$ decay of hydrogen with a fixed energy ratio $x=0.5$ between the photons. Results are shown for the initially polarized $\mu_{i}= \pm 1 / 2$ states of the atom and as a function of $\theta_{2}\left(\theta_{1}=0^{\circ}\right.$ and $\left.\varphi_{2}=0^{\circ}\right)$. Since the final polarization state of the photon pair is always pure in this case, the Bell violation and concurrence measure are equivalent.

is always pure. In more detail, we obtain the maximally entangled (Bell) state $\left|\Psi^{+}\right\rangle=\left(\left|\sigma^{+} \sigma^{-}\right\rangle+\left|\sigma^{-} \sigma^{+}\right\rangle\right) / \sqrt{2}$ if both photons are emitted in parallel $\left(\theta_{2}=0^{\circ}\right)$, and the Bell state $\left|\Phi^{+}\right\rangle=\left(\left|\sigma^{+} \sigma^{+}\right\rangle+\left|\sigma^{-} \sigma^{-}\right\rangle\right) / \sqrt{2}$ for a back-toback emission $\left(\theta_{2}=180^{\circ}\right)$. For all other geometries of the photon emission both, the concurrence and Bell violation, decrease and vanish completely if the photons are emitted perpendicular to each other $\left(\theta_{2}=90^{\circ}\right)$. As pointed out in reference [26] for the $2 s_{1 / 2} \rightarrow 1 s_{1 / 2}$ decay of hydrogen-like ions, this typical behaviour of the concurrence (and the Bell violation for a pure final state of the photon pair) can be understood as a geometrical effect due to the conservation of angular momentum. Note that Figure 2 is obtained for the special geometry of a two-photon measurement in which the emission of the 'first' photon is fixed along the direction of the quantization axis: $\theta_{1}=0^{\circ}$. In fact, this choice of the geometry coincides with those of an initially unpolarized atom where the results may depend only on the opening angle $\theta \equiv \theta_{2}$.

\subsection{The $3 \mathrm{~d}_{5 / 2} \rightarrow 1 \mathrm{~s}_{1 / 2}$ decay}

For the $3 d_{5 / 2} \rightarrow 1 s_{1 / 2}$ decay of hydrogen, the entanglement and Bell violation will not coincide in general since the polarization state of the photon pair is no longer a pure state. As shown in reference [26], moreover, the two photons are not entangled if the atoms are initially unpolarized or if only (one of) the $\mu_{i}= \pm 5 / 2$ substates is populated. In the discussion below, let us therefore restrict ourselves to the population of either the $\mu_{i}=1 / 2$ or $\mu_{i}=3 / 2$ sublevels of hydrogen excited to the $3 d_{5 / 2}$ level. In Figure 3, we first display an 'overview' about the
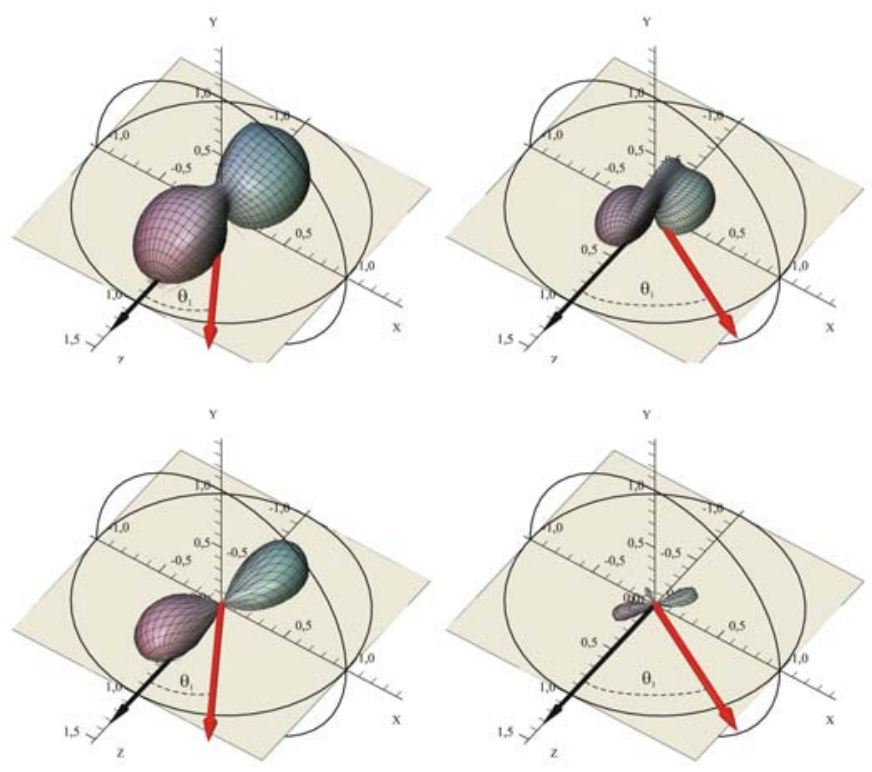

Fig. 3. (Color online) Visualization of the angular distribution of the two-photon entanglement (upper panel) and the Bell violation (lower panel) for the $3 d_{5 / 2} \rightarrow 1 s_{1 / 2}$ two-photon decay of hydrogen in the initial substate $\mu_{i}= \pm 1 / 2$. The black arrows define the $z$-axes, while the red arrows in the reaction plane indicate the direction of the first photon, i.e. $\theta_{1}=30^{\circ}$ and $\theta_{1}=60^{\circ}$ (left and right column, respectively).

angular distribution of both, the concurrence and the Bell violation, as function of the direction (i.e. the two angles $\theta_{2}$ and $\left.\varphi_{2}\right)$ under which the second photon is emitted with regard to the quantization axis. In this figure, the first photon is assumed to leave the atom under the fixed direction of either $\theta_{1}=30^{\circ}$ (left column) or $\theta_{1}=60^{\circ}$ (right column) in the $x-z$ reaction plane. For virtually all directions of the second photon, the concurrence of the two-photon polarization state (upper panel) is significantly larger than the Bell violation (lower panel). Moreover, the distribution of the entanglement and Bell violation changes from an initially dipole-like shape towards a more complicated behaviour if the emission angle $\theta_{1}$ is increased, and this change comes together with a decrease of both measures.

The clear decrease of both measures is seen also in Figure 4 where we present the concurrence and the Bell violation as functions of $\theta_{2}$, if the first photon is emitted under $\theta_{1}=30^{\circ}$ (upper panel) or $\theta_{1}=60^{\circ}$ (lower panel) as well as for different azimuth angles $\varphi_{2}=0^{\circ}, 45^{\circ}, 85^{\circ}$ of the second photon (columns 1-3). Especially for $\theta_{1}=$ $30^{\circ}$ (upper panel), the concurrence behaves qualitatively rather similar to the case of the $2 s_{1 / 2} \rightarrow 1 s_{1 / 2}$ decay: the entanglement reaches here the maximum $\left(\mathcal{C}_{\max } \approx 0.77\right)$ for $\theta_{2}=0^{\circ}$ and $\theta_{2}=180^{\circ}$, while a minimum concurrence is found for $\theta_{2}$ in the range of $60 \ldots 90^{\circ}$. Except for $\varphi_{2}=0^{\circ}$, however, the concurrence is always greater than zero for all angles.

On the other hand, the degree of Bell violation is significantly lower than the concurrence for all angles, and it vanishes completely for a large range of $\theta_{2}$ values. The maximal values of the Bell violation vary between 0.61 and 

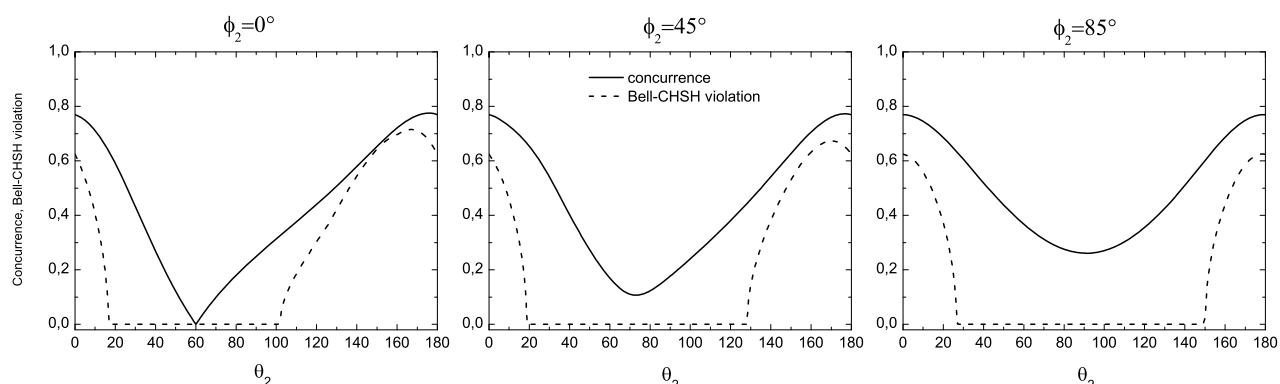

$\theta_{2}$

$\theta_{2}$
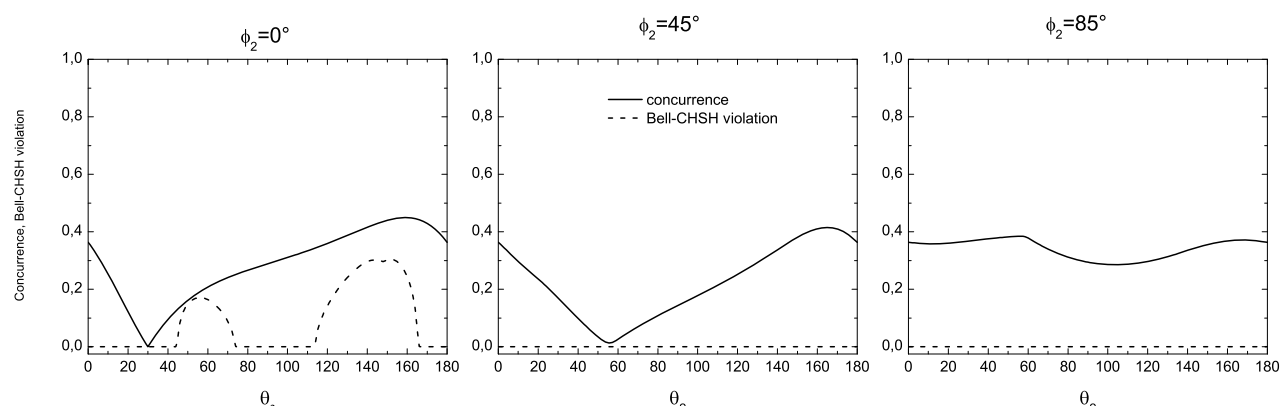

Fig. 4. Concurrence and Bell violation in the $3 d_{5 / 2} \rightarrow 1 s_{1 / 2}$ decay for an initial atomic substate $\mu_{i}= \pm 1 / 2$. Results are shown as function of $\theta_{2}$ and for fixed angles $\theta_{1}=30^{\circ}$ (upper panel), $\theta_{1}=60^{\circ}$ (lower panel) and $\varphi_{2}=0^{\circ}, 45^{\circ}, 85^{\circ}$. These curves correspond to different cuts in Figure 3.
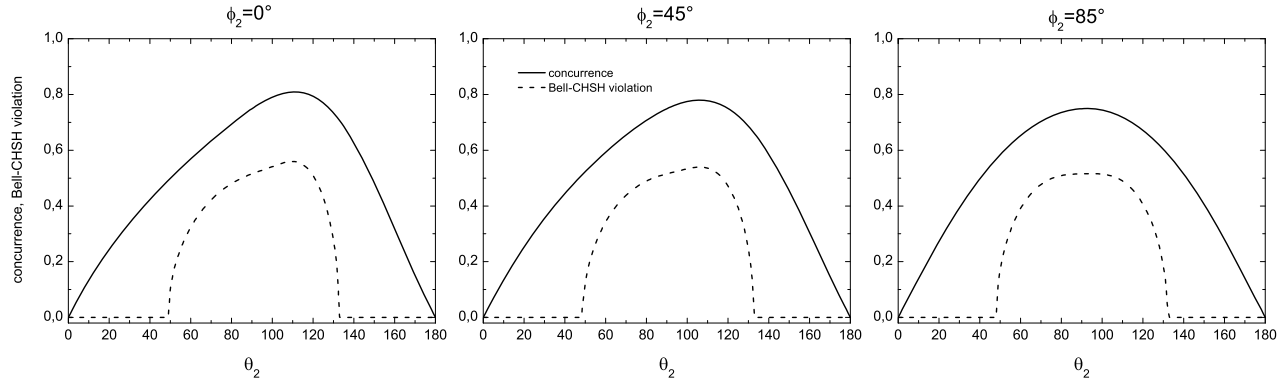

Fig. 5. Same as Figure 4 but for for the initial atomic substate $\mu_{i}= \pm 3 / 2$ and $\theta_{1}=60^{\circ}$.

0.63 , and they are located close to the concurrence maxima. As seen already from Figure 3 (right column), the difference between the concurrence and the Bell violation becomes even more apparent for $\theta_{1}=60^{\circ}$ (Fig. 4, lower panel). Although the maximum value of the concurrence decreases to $\mathcal{C}_{\max } \approx 0.42$, the Bell violation vanishes for all geometries except for $\varphi_{2}=0^{\circ}$, i.e. a violation of the Bell-CHSH inequalities occurs only for a photon emission within the reaction plane and even then only in the regions $\theta_{2} \approx 42^{\circ} \ldots 73^{\circ}$ and $\theta_{2} \approx 113^{\circ} \ldots 163^{\circ}$.

In Figure 5, finally, similar curves are shown for the population of the initial substate $\mu_{i}=3 / 2$, if the first photon is emitted under $\theta_{1}=60^{\circ}$. In this case, both the concurrence and Bell violation take their maximum values in the plane that is orthogonal to the quantization axis. This means that, independent of the azimuth angle $\varphi_{2}$, the concurrence vanishes for $\theta_{2}=0^{\circ}$ and $\theta_{2}=180^{\circ}$, while it reaches its maximum value $\left(\approx 0.8\right.$ for $\left.\theta_{1}=60^{\circ}\right)$ close to $\theta_{2}=90^{\circ}$, with the exact position varying for different values of $\varphi_{2}$. Again the Bell violation generally takes lower values ( $\approx 0.55$ at maximum) and even vanishes for $\theta_{2} \leq$ $50^{\circ}$ and $\theta_{2} \geq 132^{\circ}$. In contrast to the initial $\mu_{i}=1 / 2$ substate, the principal shape of both curves remain similar with increasing (decreasing) values of $\theta_{1}$. For $\theta_{1}<30^{\circ}$, however, there is no Bell violation, independent of the other angles of the photon emission, while a significant concurrence can still be found.

\section{Summary}

We have studied the difference between the (polarization) entanglement and the violation of the Bell-CHSH inequality for the two-photon decay of atomic hydrogen. In the $2 s_{1 / 2} \rightarrow 1 s_{1 / 2}$ decay, especially, the emitted photons end always up in a pure (composite) state and, hence, the two measures must coincide as expected from the literature.

For the $3 d_{5 / 2} \rightarrow 1 s_{1 / 2}$ case, in contrast, the final state of the emitted photon pair is generally mixed and leads to significant differences between the entanglement and the Bell violation. Apart from the decay geometry, here the results also depend on the initial polarization (substate) 
of the atom. While the two measures differ qualitatively especially for $\mu_{i}=1 / 2$, their overall shape behaves rather similar for the two-photon decay of the $\mu_{i}=3 / 2$ sublevel. These results show that the violation of the Bell-CHSH inequality is not well suited in most cases to detect entanglement, i.e. the nonlocality of the state may remain hidden. For future experiments, it seems therefore advisable to implement direct measurement schemes for the entanglement $[38,39]$. In addition, it is meanwhile known that several copies of an entangled two-qubit state can always be used in order to extract ('distill') pure-state entanglement [40-42]. In this context, it would therefore be interesting to explore the entanglement distillation properties of mixed entangled (two-photon) states that occur in the $3 d_{5 / 2} \rightarrow 1 s_{1 / 2}$ decay. Investigations in this direction are currently underway and will be reported elsewhere.

The work of A.S. was supported by the Helmholtz Gemeinschaft (Nachwuchsgruppe VH-NG-421). S.F. acknowledges support by BMBF and GSI (project No. KS-FRT).

\section{References}

1. M. Göppert-Mayer, Ann. Phys. 9, 273 (1931)

2. G. Breit, E. Teller, Astrophys. J. 91, 215 (1940)

3. S. Klarsfeld, Phys. Lett. A 30, 382 (1969)

4. C.K. Au, Phys. Rev. A 14, 531 (1976)

5. S.P. Goldman, G.W.F. Drake, Phys. Rev. A 24, 183 (1981)

6. G.W.F. Drake, S.P. Goldman, Phys. Rev. A 23, 2093 (1981)

7. J.P. Santos, F. Parente, P. Indelicato, Eur. Phys. J. D 3, 43 (1998)

8. M. Lipeles, R. Novic, N. Tolk, Phys. Rev. Lett. 15, 690 (1965)

9. R. Marrus, R.W. Schmieder, Phys. Rev. A 5, 1160 (1972)

10. P.H. Mokler, R.W. Dunford, Phys. Scr. 69, 1 (2004)

11. W. Perrie, A.J. Duncan, H.J. Beyer, H. Kleinpoppen, Phys. Rev. Lett. 54, 1790 (1985)

12. T. Haji-Hassan, A.J. Duncan, W. Perrie, H. Kleinpoppen, E. Merzbacher, Phys. Rev. Lett. 62, 237 (1989)

13. T. Haji-Hassan, A.J. Duncan, W. Perrie, H. Kleinpoppen, E. Merzbacher, J. Phys. B 24, 5035 (1991); H. Kleinpoppen, A.J. Duncan, H.-J. Beyer, Z.A. Sheikh, Phys. Scr. T 72, 7 (1997)

14. A. Aspect, J. Dalibard, G. Roger, Phys. Rev. Lett. 49, $1804(1982)$

15. J.S. Bell, Physics (Long Island City, N.Y.) 1, 195 (1964)
16. A. Einstein, B. Podolsky, N. Rosen, Phys. Rev. 47, 777 (1935)

17. E. Schrödinger, Proc. Cam. Phil. Soc. 31, 555 (1935); E. Schrödinger, Proc. Cam. Phil. Soc. 32, 446 (1936)

18. N. Bohr, Nature 136, 65 (1935); N. Bohr, Phys. Rev. 48, $696(1935)$

19. M.A. Nielsen, I.L. Chuang, Quantum Computation and Quantum Information (Cambridge Univ. Press, Cambridge, 2000)

20. M.B. Plenio, S. Virmani, Quantum Inform. Comput. 7, 1 (2007)

21. R.F. Werner, Phys. Rev. A 40, 4277 (1989)

22. J.F. Clauser, M.A. Horne, A. Shimony, R.A. Holt, Phys. Rev. Lett. 23, 880 (1969)

23. W.K. Wootters, Phys. Rev. Lett. 80, 2245 (1998)

24. A. Surzhykov, P. Koval, S. Fritzsche, Phys. Rev. A 71, 022509 (2005)

25. P. Koval, S. Fritzsche, A. Surzhykov, J. Phys. B 36, 873 (2003)

26. T. Radtke, A. Surzhykov, S. Fritzsche, Phys. Rev. A 77, 022507 (2008)

27. K. Blum, Density Matrix Theory and Applications (Plenum Press, New York, 1981)

28. V.V. Balashov, A.N. Grum-Grzhimailo, N.M. Kabachnik, Polarization and Correlation Phenomena in Atomic Collisions (Kluwer Academic, Plenum Publishers, New York, 2000)

29. S. Fritzsche, A. Surzhykov, T. Stöhlker, Phys. Rev. A 72, 012704 (2005)

30. L. Borowska, A. Surzhykov, T. Stöhlker, S. Fritzsche, Phys. Rev. A 74, 062516 (2006)

31. N. Gisin, Phys. Lett. A 154, 201 (1991)

32. R. Horodecki, Phys. Lett. A 210, 233 (1996)

33. A. Miranowicz, Phys. Lett. A 327, 272 (2004)

34. F. Verstraete, M.M. Wolf, Phys. Rev. Lett. 89, 170401 (2002)

35. S. Fritzsche, J. Electron. Specrosc. Relat. Phenom. 114116, 1155 (2001); S. Fritzsche, Phys. Scr. T 100, 37 (2002)

36. T. Radtke, S. Fritzsche, Comput. Phys. Commun. 173 (2005) 91; Comput. Phys. Commun. 175, 145 (2006)

37. T.-C. Wei, K. Nemoto, P.M. Goldbart, P.G. Kwiat, W.J. Munro, F. Verstraete, Phys. Rev. A 67, 022110 (2003)

38. P. Horodecki, Phys. Rev. Lett. 90, 167901 (2003)

39. F. Mintert, A. Buchleitner, Phys. Rev. Lett. 98, 140505 (2007)

40. N. Gisin, Phys. Lett. A 210, 151 (1996)

41. C.H. Bennett, G. Brassard, S. Popescu, B. Schumacher, J. Smolin, W.K. Wootters, Phys. Rev. Lett. 78, 2031 (1996)

42. M. Horodecki, P. Horodecki, R. Horodecki, Phys. Rev. Lett. 78, 574 (1997) 